\title{
DEPENDENCE ON THE PARTIAL PRESSURE OF OXYGEN ON THE SHIFT IN THE ENERGY BAND-GAP OF CdO THIN FILMS IN THE VISIBLE REGION
}

\author{
M. A. GRADO-CAFFARO*, M. GRADO-CAFFARO \\ and S. L. SAPIENZA ${ }^{\dagger}$ \\ C./Julio Palacios 11, 9-B, 28029-Madrid, Spain
}

(Received 5 December 2000; In final form 19 March 2001) In this paper, the dependence on the partial pressure of oxygen of the shift in the energy
band-gap of $\mathrm{CdO}$ thin films for the visible region is investigated from the theoretical
point of view on an experimental basis. In our analysis, the role played by the dependence of the carrier density upon the above pressure is emphasized.

Keywords: CdO thin films; Shift in the energy band-gap; Partial pressure of oxygen; Visible region

\section{INTRODUCTION}

It is well-known that cadmium oxide is a semiconductor that behaves as a very transparent material in the visible range. In addition, the wide band-gap of this material allows us to consider $\mathrm{CdO}$ as a semiinsulating oxide; a shift of $0.4 \mathrm{eV}$ in this band-gap has been observed (from $2.3 \mathrm{eV}$ to $2.7 \mathrm{eV}$ ) $[1,2]$. Certainly, the relevance of this shift is manifest in order to perform a deep analysis of the physical behaviour of the material in question with respect to the partial pressure of oxygen involved in the growth of CdO crystals [1-3]. At this point, we

${ }^{*}$ Corresponding author.

${ }^{\dagger}$ Scientific consultant. 
can mention that in Ref. [2] an experimental study on the relevance of the above pressure was carried out; in fact, within this study, cadmium oxide thin films were prepared by evaporating cadmium in the presence of oxygen and the effects of varying the partial pressure of oxygen were discussed. These films presented the n-type conductivity in the margin of the above pressure utilized. On the other hand, in Ref. [2] experimental results referring optical transmittance in the visible region were reported; the dependence of this transmittance on the oxygen partial pressure during the deposition process was significant [2].

The aim of the present paper consists of to investigate the dependence on the partial pressure of oxygen of the shift experienced by the band-gap of cadmium oxide in the visible region. In this study, the dependence of this shift on carrier density plays an important role. In addition, we shall consider the coefficient of optical absorption in terms of the partial pressure of oxygen for a given photon energy. At this point, it is important to remark that the shift in the optical absorption coefficient as a consequence of the shift in the energy band-gap has been studied in Ref. [4] for the ultraviolet range and in Ref. [5] for the visible one. In particular, Ref. [5] offers a quantitative analysis in which the main results refer to the average coefficient of optical absorption for the upper quarter of the visible region and to the average shift of this coefficient for the same wavelength margin. Furthermore, it is important to remark that both Refs. [4] and [5] are studies for the amorphous phase of the material in question.

\section{THEORY}

First of all, we shall write down the mathematical expression that gives the shift in the energy band-gap observed in a number of transparent oxides (in particular, here we consider $\mathrm{CdO}$ ); this expression can be found, for example, in Refs. [4,5] and is as follows:

$$
\Delta E_{g}=\frac{\hbar^{2}}{2 m_{r}^{*}} \cdot\left(3 \pi^{2} n\right)^{2 / 3}
$$


where $\hbar$ is the reduced Planck's constant, $\mathrm{m}_{\mathrm{r}}^{*}$ is the reduced effective mass of an electron-hole pair namely $m_{r}^{*}=m_{n}^{*} m_{p}^{*} /\left(m_{n}^{*}+m_{p}^{*}\right), m_{n}^{*}$ and $m_{p}^{*}$ being the effective masses of electrons and holes respectively, and $\mathrm{n}$ stands for electron density ( $\mathrm{n}$-type $\mathrm{CdO}$ is considered).

The electron density depends on the partial pressure of oxygen so that we can consider a function $n(P)$ where $P$ denotes partial pressure of oxygen; in Figure 1 an experimental plot of this function is represented [2]. In this figure, it is easy to observe that for values of the pressure in question $\mathrm{P} \lesssim 1 \times 10^{-3}$ torr, carrier density is increasing with $\mathrm{P}$; on the other hand, $\mathrm{n}$ decreases for $\mathrm{P} \gtrsim 1 \times 10^{-3}$ torr [2] (the value $P=1 \times 10^{-3}$ torr can be denominated optimum pressure because, at this pressure, optical transmittance achieves the maximum value which in the study of Ref. [2] was $90 \%$ ). By taking into account the above considerations, we may rewrite Eq. (1) as follows:

$$
\Delta \mathrm{E}_{\mathrm{g}}(\mathrm{P})=\frac{\hbar^{2}}{2 \mathrm{~m}_{\mathrm{r}}^{*}} \cdot\left[3 \pi^{2} \mathrm{n}(\mathrm{P})\right]^{2 / 3}
$$

so that, differentiating Eq. (2), we get:

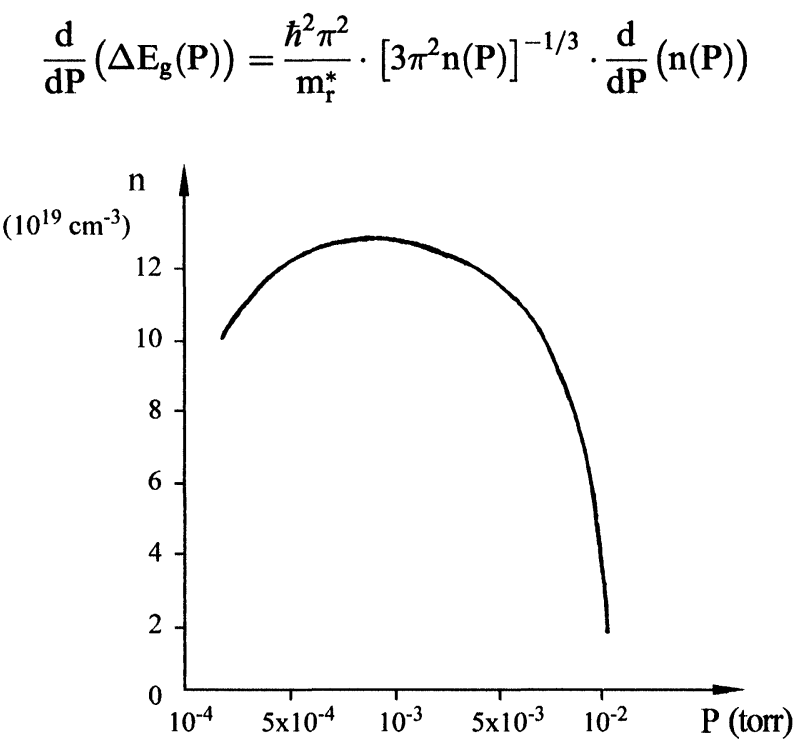

FIGURE 1 Variation of electron density against partial pressure of oxygen (after Ref. [2]). 
Therefore, inspecting formula (3), it is very easy to see that for $\mathrm{P} \lesssim 1 \times 10^{-3}$ torr, $\left(\mathrm{d}\left(\Delta \mathrm{E}_{\mathrm{g}}(\mathrm{P})\right) /(\mathrm{dP})>0\right)$ since $(\mathrm{dn} / \mathrm{dP})>0$ (this situation corresponds to amorphous state $[4,5])$; in addition, by considering also formula (3), for $P \gtrsim 1 \times 10^{-3}$ torr, since $(\mathrm{dn} / \mathrm{dP})<0$, it follows that $\left(d\left(\Delta E_{g}(P)\right) /(d P)<0\right)$ (this situation corresponds to crystalline state $[4,5])$.

Next we shall consider the following expression for the optical absorption coefficient valid for CdO (see, for example, Ref. [6]):

$$
\alpha(\omega ; \mathbf{P})=\mathbf{A} \cdot \frac{\left[\hbar \omega-\mathrm{E}_{\mathbf{g}}(\mathbf{P})\right]^{1 / 2}}{\hbar \omega}
$$

where $\mathrm{A}$ is a positive real constant and $\hbar \omega$ is the photon energy; notice that in Eq. (4) P acts as a parameter. In fact, Figure 3 of Ref. [2] shows a family of curves taking $\hbar \omega$ as abscissa and $(\alpha \hbar \omega)^{2}$ as ordinate; in other words, we consider a geometrical interpretation by which a parameter (in our case, the oxygen partial pressure P) defines a set of curves so that, for a given fixed value of this parameter, we have a concrete curve representing $(\alpha \hbar \omega)^{2}$ as a function of $\hbar \omega$. At this point, we must remark that we have performed calculations tending to extrapolate these curves so that a set of curves passing by the point $\left(2 \mathrm{eV}, 0.01\left(\mathrm{eV}^{2} / \mathrm{cm}^{2}\right)\right)$ has been obtained. However, it is clear that this extrapolation is not consistent with Eq. (4) since the condition $\hbar \omega \geq \mathrm{E}_{\mathrm{g}}$ is not fulfilled.

Finally, fixing, $\omega$, let us differentiate Eq. (4) as a function of P; we have:

$$
\Delta \alpha=-\mathrm{A} \cdot \frac{\mathrm{dE}_{\mathrm{g}}(\mathrm{P}) / \mathrm{dP}}{2 \hbar \omega\left[\hbar \omega-\mathrm{E}_{\mathrm{g}}(\mathrm{P})\right]^{1 / 2}} \cdot \Delta \mathrm{P}
$$

With $\Delta \alpha<0$ (see Eq.(3) of Ref. [5]) and ( $\left.\mathrm{dE}_{\mathrm{g}} / \mathrm{dP}\right) \lessgtr 0$ for amorphous and crystalline states respectively (for more details related to this, see Ref. [4]) so that, by virtue of the previous discussion, it follows that $\Delta \mathbf{P}<0$ for amorphous state and $\Delta \mathbf{P}>0$ for crystalline state. The relevance of the shift $\Delta \alpha$ is clearly manifest $[4,5]$ and, on the other hand, we can claim that further studies related to this shift and the role played by $\mathbf{P}$ should be very useful to explain theoretically some facts that have been investigated experimentally with limited success. 


\section{CONCLUSIONS}

We have analyzed the optical behaviour of $\mathrm{CdO}$ thin films in the visible range with respect to the partial pressure of oxygen during the deposition process in the context of the technique of activated reactive evaporation. In this analysis, results concerning the variation of the band-gap shift with the partial pressure of oxygen have been reported as well as $\Delta \alpha$ versus $\Delta \mathrm{P}$; these results refer to both crystalline and amorphous phases. In particular, with respect to the shift $\Delta \alpha$, it is important to notice that $\Delta \mathbf{P}$ should be taken with respect to the optimal value $P_{0}=1 \times 10^{-3}$ torr as reference value, that is to say, $\Delta P=P-P_{0}$ where $P_{0}=1 \times 10^{-3}$ torr and $P$ designates a generic value of oxygen partial pressure. On the other side, the relevance of the correlation between $\mathbf{n}$ and $\mathbf{P}$ is remarkable.

\section{References}

[1] Lal Chopra, K. and Ranjan Das, S., Thin film solar cells (Plenum Press, New York, 1983), p. 325.

[2] Sravani, C., Ramakrishna Reddy, K. T. and Jayarama Reddy, P. (1993). Mater. Lett., 15, 356-358.

[3] Kuku, T. A. (1986). Thin Solid Films, 142, 241.

[4] Grado-Caffaro, M. A. and Grado-Caffaro, M. (1995). Mod. Phys. Lett. B, 9, $1631-1634$.

[5] Grado-Caffaro, M. A. and Grado-Caffaro, M. (1999). Optik, 110, 395-396.

[6] Pankove, J. I., Optical processes in semiconductors (Dover, New York, 1971). 

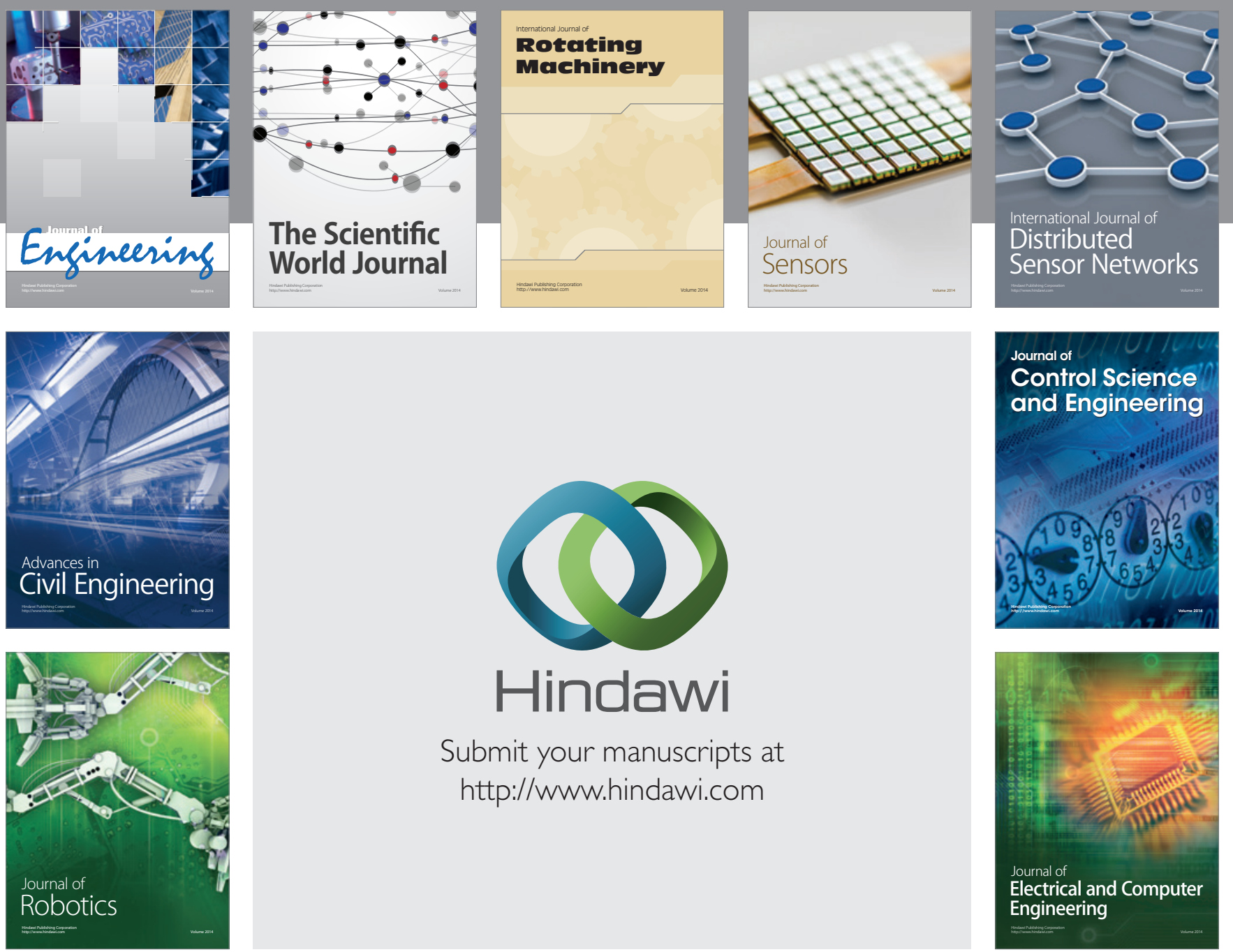

Submit your manuscripts at

http://www.hindawi.com
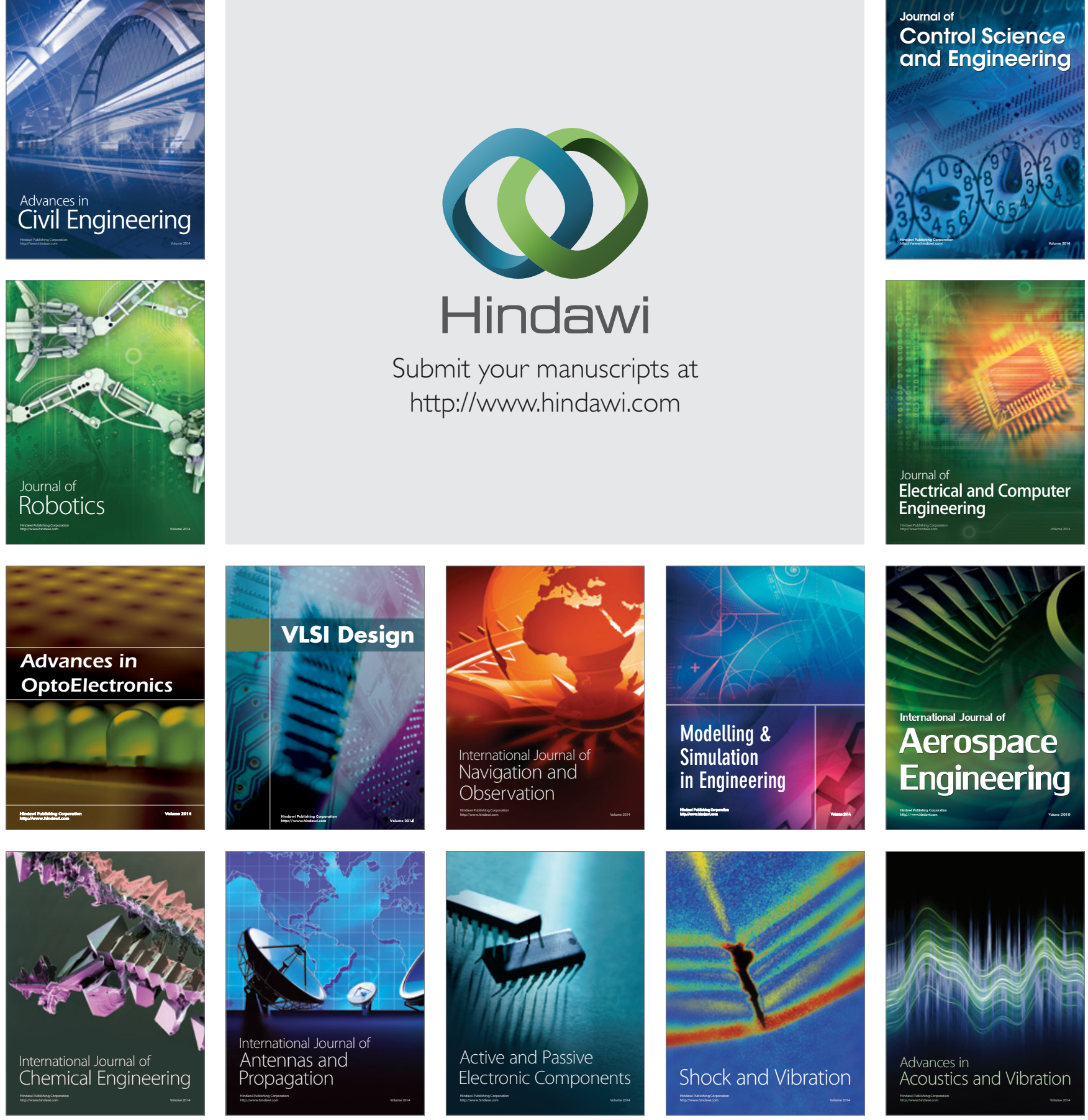